\title{
ANÁLISE DA VARIAÇÃO DA SOLUBILIDADE DE ALFA- TOCOFEROL COM A TEMPERATURA EM MISTURA ETANOL+ÁGUA
}

\author{
L. G. FONSECA ${ }^{1}$, J. B. RODRIGUES ${ }^{1}$, C. V. da SILVA $^{1}$ e R. A. MALAGONI ${ }^{1}$ \\ ${ }^{1}$ Universidade Federal de Uberlândia, Faculdade de Engenharia Química \\ E-mail para contato: malagoni@feq.ufu.br
}

\begin{abstract}
RESUMO - O alfa-tocoferol, mais conhecido como vitamina E, é um composto lipossolúvel, sendo necessária a adição de outro solvente que propicie um ambiente mais hidrofóbico para aumentar a solubilidade do mesmo. Neste trabalho, o solvente escolhido foi o etanol nas quantidades de 10 e $40 \% \mathrm{~m} / \mathrm{m}$. A faixa de temperatura estudada foi de 20 a $40^{\circ} \mathrm{C}$. Nos ensaios de solubilidade, utilizou-se uma célula de equilíbrio de vidro borossilicato encamisada acoplada a um banho termostatizado. A agitação da mistura foi realizada por uma barra magnética revestida com teflon durante um período de $24 \mathrm{~h}$. Na sequência, o sistema permaneceu em repouso por $2 \mathrm{~h}$, para então serem coletadas três amostras, sendo mensuradas as absorbâncias para calcular a solubilidade da vitamina $\mathrm{E}$ em diferentes temperaturas com o uso das curvas de calibração previamente construídas.
\end{abstract}

\section{INTRODUÇÃO}

A vitamina $\mathrm{E}$, é um composto lipossolúvel que apresenta oito diferentes formas sendo que, quatro delas são os tocoferóis ( $\alpha-, \gamma-, \beta-$ e $\delta$-) e quatro tocotrienóis ( $\alpha-, \gamma-, \beta-$ e $\delta$-) (Zingg, 2007). Desses compostos, o alfa-tocoferol é considerado o mais antioxidante (Di Mascio et al., 1991) e o que possui a mais alta retenção em animais (Schneider, 2005). A vitamina E posssui grande importância para a saúde humana sendo considerado um nutriente essencial para a formação e a manutenção ideal da integridade do sistema nervoso humano (Sokol, 1998).

O $\alpha$-tocoferol é formado por uma extensa cadeia de carbonos que possui um grupo éter e um grupo fenol. Apesar de esses dois grupos serem capazes de formar ligações de hidrogênio com a água, a cadeia de alcanos apresenta tamanho suficiente fazendo com que a molécula possua um caratér apolar sendo, portanto, pouco solúvel em água. Devido a essa baixa solubilidade em água torna-se necessária a adição de outro solvente. A adição do etanol propicia um ambiente mais hidrofóbico e aumenta a solubilidade da vitamina E.

Neste trabalho, o solvente escolhido foi o etanol nas quantidades de 10 e $40 \% \mathrm{~m} / \mathrm{m}$. A faixa de temperatura estudada foi de 20 a $40^{\circ} \mathrm{C}$, sendo usada uma célula de equilíbrio de vidro borossilicato. A mistura foi agitada por $24 \mathrm{~h}$. Após $2 \mathrm{~h}$ de repouso, realizaram-se amostragens, a fim de determinar a solubilidade no espectrofotômetro. 


\section{MATERIAL E MÉTODOS}

Nos ensaios de solubilidade foi utilizada uma célula de equilíbrio de vidro borossilicato, encamisada e com capacidade de $40 \mathrm{~mL}$. Um banho termostatizado (Marconi, MA-184) manteve a temperatura do sistema constante, sendo que a mesma foi mensurada com o uso de um termopar calibrado ligado a um indicador de temperatura (Full Gauge Controls, TIC 17RGTi). O termopar foi inserido na célula de forma que fosse possível acompanhar a temperatura da solução durante todo o experimento. Um agitador magnético (IKA, RH D-KT/C) e uma barra magnética $(1,512 \mathrm{~cm}$ de comprimento e $0,607 \mathrm{~cm}$ de diâmetro) revestida com teflon foram utilizados para promover a homogeneização da mistura que consistia em um período de $24 \mathrm{~h}$ de agitação seguidas por $2 \mathrm{~h}$ de repouso. Esse tempo foi escolhido, pois dentre os experimentos realizados por Oliveira (2013), para se analisar a influência dos tempos de agitação e repouso na solubilidade da vitamina E, estes tempos apresentaram resultados constantes de solubilidade, o que comprovou a saturação do meio.

No procedimento experimental, primeiro colocava-se o alfa-tocoferol em excesso e, $\operatorname{logo}$ em seguida, a solução de etanol+água. $\mathrm{O}$ alfa-tocoferol utilizado nesse trabalho consistia em uma mistura racêmica de seus isômeros ópticos, da marca SIGMA com uma pureza de 95\% e o etanol utilizado foi da marca Proquímios e possui uma pureza de 99,8\%. A água destilada e deionizada utilizada foi obtida pelo próprio laboratório e possui um $\mathrm{pH}=6,48$. A quantidade de água presente no etanol foi analisada e levada em consideração neste trabalho.

Para cada uma das temperaturas estudadas três amostras foram retiradas e suas respectivas absorbâncias foram medidas através do uso de um espectrofotômetro (T60, UV-SpectrophotometerPG Instruments). O ponto de amostragem se localizava no meio da célula de equilíbrio e as amostras eram retiradas de forma cuidadosa para que não houvesse a passagem de sólidos para a seringa. Caso isso ocorresse, a amostra era descartada e a seringa era lavada antes de se coletar outra. Para trabalhar com a vitamina $\mathrm{E}$, dois comprimentos de onda podem ser utilizados, sendo eles, 291,6 e $300 \mathrm{~nm}$. Neste trabalho, optou-se pelo comprimento de onda de $291,6 \mathrm{~nm}$, por ser o mesmo utilizado por Dubbs e Gupta (1998).

\subsection{Unidade Experimental}

A unidade experimental utilizada está representada na Figura 1.

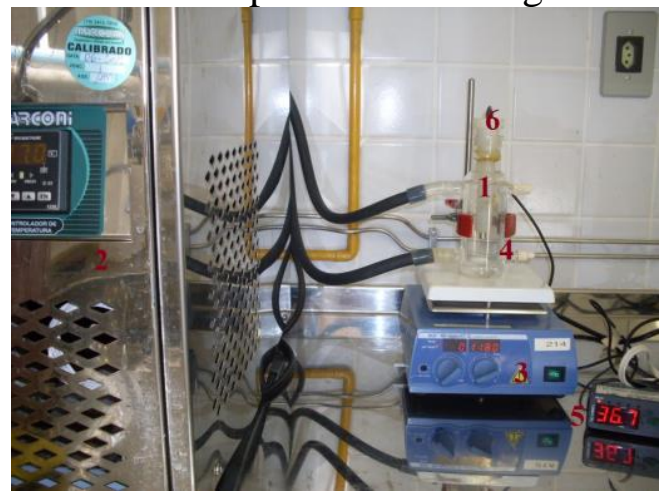

Figura 1 - Unidade Experimental. 
Na Figura 1, pode-se observar que (1) é a célula de equilíbrio, (2) é o banho termostatizado, (3) o agitador magnético, (4) a barra magnética, (5) o indicador de temperatura e (6) a rolha de tecnyl que suporta o termopar.

\subsection{Curva de Calibração}

Para que fosse possível a análise da concentração de vitamina E através da absorbância foi necessária a construção de uma curva de calibração. O método utilizado para a construção dessa curva consistiu em solubilizar previamente a vitamina E no etanol com o auxílio de uma espátula e depois adicionava-se a água. Essa prática garante uma solubilização mais eficiente da vitamina e evita a perda de etanol por evaporação. Após isto, a mistura foi inserida em uma célula de vidro borossilicato, encamisada, na qual ela ficou em agitação por um período de $24 \mathrm{~h}$, através do uso de um agitador magnético (IKA, RH D-KT/C) e de uma barra magnética revista com teflon $(1,512 \mathrm{~cm}$ de comprimento e $0,607 \mathrm{~cm}$ de diâmetro). Na Figura 2, é possível observar a célula utilizada no preparo dessa mistura.

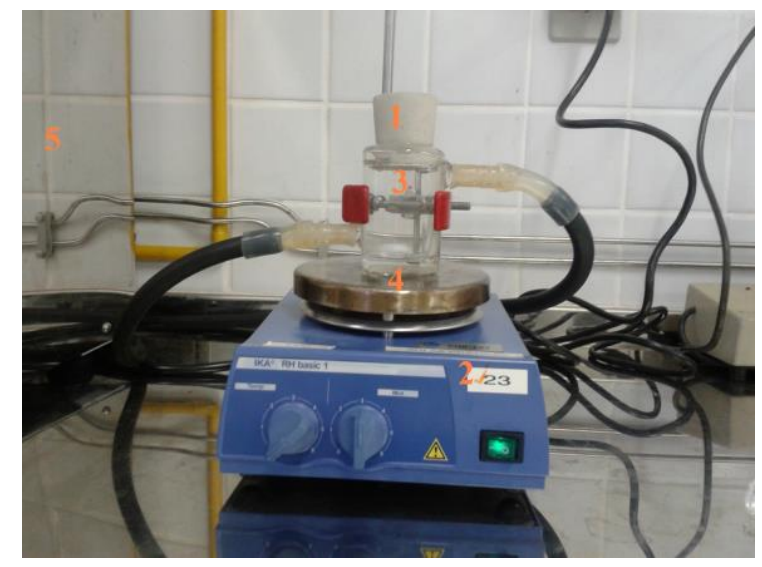

Figura 2 - Célula usada na construção da curva de calibração.

Observando a Figura 2, pode-se ver que (1) é a rolha de borracha, (2) é o agitador magnético, (3) a célula de vidro encamisada, (4) a barra magnética e (5) o banho termostatizado.

Depois de a solução passar por esse período de agitação, foram feitas diversas diluições da mesma de modo que fosse possível calcular a massa de vitamina E em cada uma delas. Em seguida, as absorbâncias de cada diluição foram medidas com o uso do espectrofotômetro (T60, UVSpectrophotometer-PG Instruments). Com esses dados e o uso do programa Statistica foi possível a obtenção das curvas de calibração para as soluções de 10 e $40 \%$ m/m de etanol.

Curva 10\% de etanol : A solução utilizada na obtenção dos dados para a curva de calibração da solução contendo $10 \%$ de etanol continha $4 \mathrm{~g}$ de etanol, $36 \mathrm{~g}$ de água destilada e deionizada e $0,013 \mathrm{~g}$ de vitamina E. 
Curva 40\% de etanol : A solução utilizada na obtenção dos dados para a curva de calibração da solução contendo $40 \%$ de etanol continha $14 \mathrm{~g}$ de etanol, $21 \mathrm{~g}$ de água destilada e deionizada e $0,048 \mathrm{~g}$ de vitamina $\mathrm{E}$.

\subsection{Ajustes de Solubilidade}

Para ajustar os dados de solubilidade encontrados neste trabalho, em função da temperatura, foi utilizada a equação empírica desenvolvida por Lee e Lahti (1972) que está representada nesse trabalho pela Equação 1.

$$
\ln S=A+B T
$$

sendo: $S$ é a solubilidade (g vit. E/g sç), $A$ e $B$ são os parâmetros da equação e $T$ a temperatura em Kelvin.

\section{RESULTADOS E DISCUSSÃO}

\subsection{Curvas de Calibração}

A Tabela 1 mostra os dados utilizados para a construção da curva de calibração, $10 \% \mathrm{~m} / \mathrm{m}$ de etanol, que está representada na Figura 3.

Tabela 1- Dados da Curva de Calibração, $10 \%$ (m/m) de etanol.

\begin{tabular}{ccc}
\hline $\begin{array}{c}C_{\text {solução concentrada }} \\
(\%)\end{array}$ & $\begin{array}{c}S \times 10^{6} \\
\text { (g vit/g solução) }\end{array}$ & $A$ \\
\hline 1,0 & 3,249 & 0,063 \\
4,6 & 14,940 & 0,156 \\
7,5 & 24,367 & 0,231 \\
10,0 & 32,489 & 0,338 \\
15,0 & 48,734 & 0,461 \\
17,5 & 56,856 & 0,554 \\
20,0 & 64,978 & 0,620 \\
25,0 & 81,223 & 0,758 \\
27,5 & 89,346 & 0,848 \\
30,0 & 97,468 & 0,902 \\
32,5 & 105,590 & 0,983 \\
\hline
\end{tabular}

A curva forneceu a equação de solubilidade da vitamina E para esta concentração de etanol, que é a Equação 2, onde $S$ representa a solubilidade da vitamina E e $A$ representa a absorbância. O coeficiente de correlação quadrático foi de 0,9995 .

$$
A=0,0274+9064,1434 S
$$




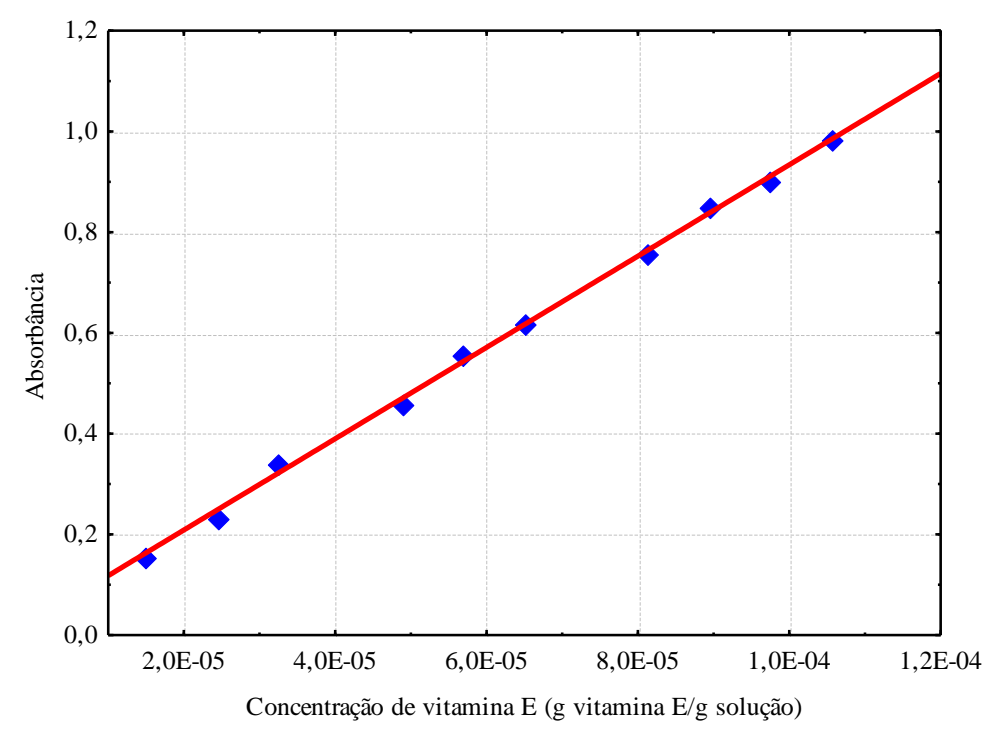

Figura 3 - Curva de Calibração, $10 \% \mathrm{~m} / \mathrm{m}$ de etanol.

Os dados usados para a construção da curva de $40 \% \mathrm{~m} / \mathrm{m}$ de etanol estão descritos na Tabela 2 .

Tabela 2 - Dados da Curva de Calibração, $40 \% \mathrm{~m} / \mathrm{m}$ de etanol.

\begin{tabular}{ccc}
\hline $\begin{array}{c}C_{\text {solução concentrada }} \\
(\%)\end{array}$ & $\begin{array}{c}S \times 10^{5} \\
(\mathrm{~g} \text { vit/g solução) }\end{array}$ & $A$ \\
\hline 1,0 & 1,369 & 0,068 \\
2,0 & 2,739 & 0,135 \\
3,0 & 4,109 & 0,200 \\
4,5 & 6,163 & 0,379 \\
5,0 & 6,848 & 0,414 \\
5,5 & 7,532 & 0,511 \\
7,0 & 9,587 & 0,629 \\
8,0 & 10,956 & 0,744 \\
9,0 & 12,326 & 0,852 \\
10,0 & 13,695 & 0,900 \\
10,5 & 14,380 & 0,984 \\
\hline
\end{tabular}

E a curva de calibração obtida está representada na Figura 4. 


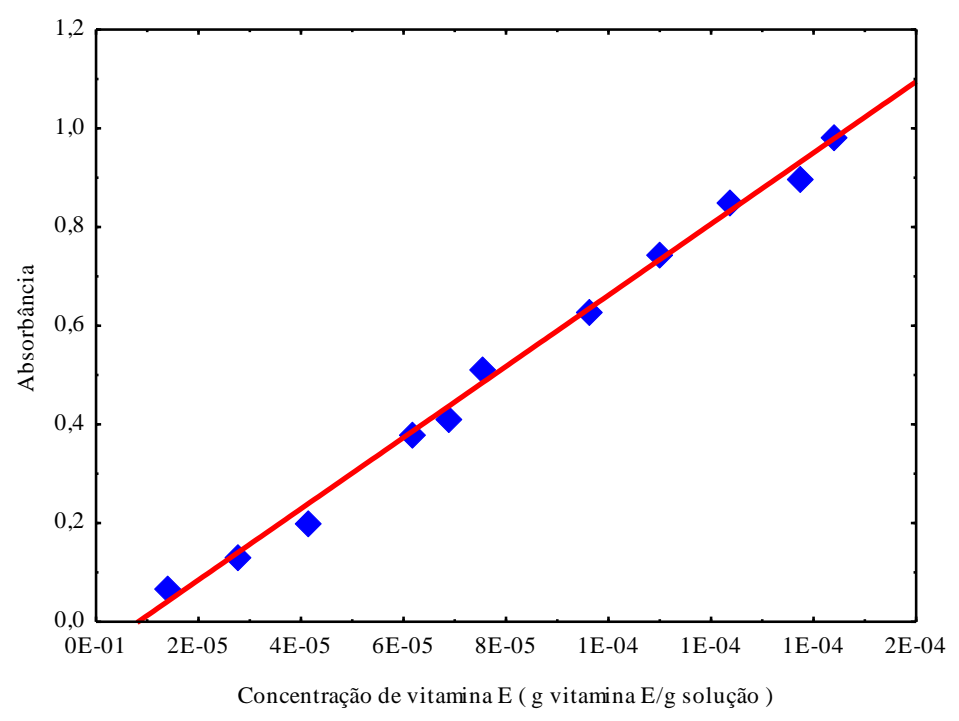

Figura 4 - Curva de Calibração, $40 \%$ m/m de etanol.

O coeficiente de correlação quadrático foi de 0,9952 e a equação que relaciona a solubilidade $(S)$ e a absorbância $(A)$ é dada na Equação 3.

$$
A=-0,0595+7213,2591 S
$$

Com os tempos de agitação e repouso já fixados ( $24 \mathrm{~h}$ de agitação e $2 \mathrm{~h}$ de repouso) e as curvas de calibração construídas foi possível encontrar a solubilidade da vitamina E para cada uma das temperaturas estudadas.

\subsection{Experimentos com $10 \%$ de etanol}

Para a concentração de $10 \%$ foram feitos experimentos nas temperaturas de $20,25,30$ e $33^{\circ} \mathrm{C}$. Em cada experimento, a vitamina $\mathrm{E}$ foi colocada em excesso na célula e, logo em seguida, colocavase a solução etanol+água. O sistema ficou em agitação por 24 h e em repouso por 2 h, sendo que após esse período foram coletadas três amostras, que logo em seguida foram analisadas através da espectrofotometria. Com a média aritmética das absorbâncias das amostras foi possível calcular a solubilidade da vitamina E através da Equação 2. Os resultados obtidos estão descritos na Tabela 3.

Tabela 3 - Solubilidade da vitamina E em função da temperatura (10\% etanol).

\begin{tabular}{cc}
\hline$T \pm \delta\left({ }^{\circ} \mathrm{C}\right)$ & $S \times 10^{5}$ (g vit./g solução) \\
\hline $20,1 \pm 0,1$ & $3,8276 \pm 0,1506$ \\
$25,0 \pm 0,1$ & $7,3248 \pm 0,0951$ \\
$30,1 \pm 0,1$ & $8,1298 \pm 0,0701$ \\
$33,1 \pm 0,1$ & $9,0643 \pm 0,0584$ \\
\hline
\end{tabular}


Para os experimentos de $10 \% \mathrm{~m} / \mathrm{m}$ de etanol, o parâmetro $A$ obtido foi significativo, já o parâmetro $B$ ultrapassou o nível de significância estabelecido ficando um pouco acima de $5 \%$ com $6,38 \%$, o que não invalida o ajuste. $\mathrm{Na}$ Tabela 4 , podem-se observar os valores dos parâmetros, o $\mathrm{R}^{2} \mathrm{e}$ o erro padrão do parâmetro. Com esses valores, nota-se que o modelo de Lee e Lahti (1972) representou bem os experimentos de $10 \%$.

Tabela 4 - Parâmetros obtidos no ajuste de solubilidade (10\% etanol).

\begin{tabular}{ccc}
\hline Parâmetro & Erro Padrão do Parâmetro & $\mathrm{R}^{2}$ \\
\hline$A=-25,9667$ & 4,3798 & 0,9480 \\
$B=0,0546$ & 0,0145 & 2945 \\
\hline
\end{tabular}

\subsection{Experimentos com $40 \%$ de etanol}

As temperaturas estudadas para a concentração de $40 \%$ de etanol foram: $25,30,33$ e $40^{\circ} \mathrm{C}$. A Equação 3 foi utilizada para calcular a solubilidade da vitamina $\mathrm{E}$ e os resultados estão descritos na Tabela 5.

Tabela 5 - Solubilidade da vitamina E em função da temperatura (40\% etanol).

\begin{tabular}{cc}
\hline$T \pm \delta\left({ }^{\circ} \mathrm{C}\right)$ & $S \times 10^{4}$ (g vit./g solução) \\
\hline $25,1 \pm 0,1$ & $7,9784 \pm 0,0764$ \\
$30,0 \pm 0,1$ & $8,6993 \pm 0,1179$ \\
$33,1 \pm 0,1$ & $9,2677 \pm 0,2590$ \\
$40,1 \pm 0,1$ & $12,6642 \pm 0,8131$ \\
\hline
\end{tabular}

Para um nível significância de 5\% no software Statistica, os experimentos de $40 \%$ de etanol obtiveram parâmetros $A$ e $B$ significativos e ao se observar o $\mathrm{R}^{2}$ e o erro padrão do parâmetro, pode-se ver que o modelo de Lee e Lahti (1972) representou bem os dados de solubilidade. Na Tabela 6 é possível observar os valores dos parâmetros, $\mathrm{o} \mathrm{R}^{2} \mathrm{e}$ o erro padrão do parâmetro.

Tabela 6 - Parâmetros obtidos no ajuste de solubilidade (40\% etanol).

\begin{tabular}{ccc}
\hline Parâmetro & Erro Padrão do Parâmetro & $\mathrm{R}^{2}$ \\
\hline$A=-17,1467$ & 1,5943 & 0,9758 \\
$B=0,0334$ & 0,0052 & 0 \\
\hline
\end{tabular}

\section{CONCLUSÃO}

Através dos experimentos realizados neste trabalho, pode-se concluir que a solubilidade da vitamina E aumenta consideravelmente com o aumento da concentração de etanol na solução. Nota-se também um crescimento na solubilidade da vitamina $\mathrm{E}$ com o aumento da temperatura. Devido aos pequenos desvios obtidos neste trabalho, verifica-se que o método e os equipamentos utilizados foram 
eficientes.

O ajuste utilizado apresentou bons resultados, sendo muito satisfatório para a concentração de $40 \%$ de etanol e relacionou bem os dados para a concentração de $10 \%$ de etanol.

\section{AGRADECIMENTOS}

Agradecemos ao Conselho Nacional de Desenvolvimento Científico e Tecnológico pela bolsa de iniciação em desenvolvimento tecnológico e inovação e também a Faculdade de Engenharia Química da Universidade Federal de Uberlândia pelo incentivo a pesquisa e estrutura física oferecida para o desenvolvimento desta pesquisa no Laboratório de Cristalização. Agradecemos também a Fundação de Amparo à Pesquisa do Estado de Minas Gerais (FAPEMIG) pelos recursos concedidos no Projeto de Participação Coletiva em Eventos Técnicos-Científicos (PCE-00082-14).

\section{REFERÊNCIAS}

DI MASCIO, P.; MURPHY, M. E.; SIES, H. Antioxidant defense systems: the role of carotenoids, tocopherols and thiols. Am. J. Clin. Nutr., v.53, p. 194-200, 1991.

DUBBS, M. D.; GUPTA, R. B. Solubility of vitamin E ( $\alpha$-tocopherol) and vitamin $\mathrm{K}_{3}$ (menadione) in ethanol-water mixture. J. Chem. Eng. Data, v. 43, p. 590-591, 1998.

LEE, F. M.; LAHTI, L. E. Solubility of urea in water-alcohol mixtures. J. Chem. Eng. Data, v. 17, p. 304-306, 1972.

OLIVEIRA, F. Solubilidade da Vitamina E ( $\alpha$-tocoferol) em misturas de etanol+água. 19 p.

Relatório Final de Iniciação Científica PIBITI, Faculdade de Engenharia Química, Universidade Federal de Uberlândia, Uberlândia, 2013.

SCHNEIDER, C. Chemistry and biology of vitamin E. Mol. Nutr. Food Res. v. 49, p. 7-30, 2005.

SOKOL, R.J. Vitamin E and neurologic function in man. Free Radical Biol. Med., v.6, p. 189-207, 2007.

ZINGG, J. M. Vitamin E: An overview of major research directions. Mol. Aspects Med., v. 28, p. 400422, 2007. 